Rev. Elev. Méd. vét. Pays trop., 1977, 30 (2) : 191-198.

\title{
Essais de régénération mécanique de quelques parcours sahéliens dégradés
}

\author{
par B. TOUTAIN (*)
}

\begin{abstract}
RÉSUMÉ
Une dégradation importante de la végétation sahélienne, surtout depuis la sécheresse, se manifeste dans certaines régions du Nord de la Haute-Volta, très chargées en bétail, entraînant la diminution des potentialités pastorales et menaçant le milieu lui-même. Les principales formes de cette dégradation ont été analysées. La régénération de terrains dènudés a été essayée dans différentes conditions, soit par des travaux superficiels du sol, soit par un sous-solage, de façon à améliorer le bilan hydrique et permettre la réinstallation d'un couvert végétal.
\end{abstract}

\section{INTRODUCTION}

Le voyageur traversant la région sahélienne de Haute-Volta ne peut manquer de remarquer qu'en certains endroits, les arbres sont en train de mourir en grandes quantités et que l'herbe a pratiquement disparu sur de larges surfaces, laissant le sol nu exposé à l'érosion. La dégradation de la végétation et du milieu est assez récente et s'est beaucoup accentuée avec la sécheresse. Le phénomène est inquiétant à plus d'un titre lorsque l'on connaît la rigueur et les irrégularités habituelles du climat et que l'on sait que l'élevage tirant essentiellement parti de la végétation naturelle représente la principale ressource de cette région, en assurant notamment une grande partie de l'alimentation de ses habitants. Le potentiel fourrager que représente cette végétation disparue est considérable et la charge du bétail se trouve reportée sur la végétation pâturable restante. La question se pose donc de savoir comment freiner rapidement la progression de la dégradation et comment aider la nature à reconstituer la végétation. L'analyse des mécanismes de la

(*) I. E. M. V. T., Service d'Agropastoralisme, 10, rue Pierre-Curie, 94700 Maisons-Alfort. dégradation a permis dans une certaine mesure, de remonter aux causes. Divers essais de restauration par des moyens mécaniques ouvrent, malgré des résultats encore modestes, quelques perspectives d'intervention.

\section{LE MILIEU}

La partie sahélienne de la Haute-Volta reçoit annuellement $350 \mathrm{~mm}$ de pluie à l'extrême Nord et $550 \mathrm{~mm}$ à Dori, répartis sur $3 \mathrm{mols}$, de mi-juin à mi-septembre. La végétation est sahélienne et sud-sahélienne. Ce sont les groupements végétaux xérophiles des hauts de versants et des pentes qui montrent le plus de fragilité à la dégradation :

- les ensablements dunaires sont couverts par une steppe herbeuse ou arbustive à tapis herbacé continu avec les graminées annuelles Schoenefeldia gracilis, Aristida mutabilis, Cenchrus biflorus et les espèces arbustives Combretum glutinosum ou Guiera senegalensis ;

- les glacis sableux ou limono-sableux sont recouverts d'une steppe arbustive très ouverte avec un tapis graminéen discontinu à base de Schoenefeldia gracilis et des arbustes comme Acacia laeta, A. tortilis ou Balanites aegyptiaca ; 
- les forêts basses sur sols squelettiques à Pterocarpus lucens sont riches en espèces ligneuses. Le tapis herbacé est discontinu et se compose principalement des graminées Schoenefeldia gracilis et Aristida adscensionis.

\section{LES FORMES DE DÉGRADATION}

Dans l'ensemble, la dégradation du milieu végétal suit partout les mêmes étapes. Elle se manifeste d'abord par une évolution de la flore herbacée et l'on note généralement le remplacement de certaines graminées par des espèces herbacées à cycle court ou réputées envahissantes. A un stade plus avancé, il y a disparition progressive du tapis herbacé avec dénudation du sol par plages, apparition ou augmentation importante du ruissellement, érosion avec déflation, transport des particules de sol et dépôt dans les parties basses. Finalement, on observe la mort des ligneux. Ce schéma ne constitue pas forcément une disparition mais plutôt une étape dans un processus de remplacement du type actuel de végétation par un autre, mieux adapté à de nouvelles conditions et la colonisation spontanée de sols dénudés a été parfois observée (1).

Dans le détail, les manifestations de la dégradation diffèrent selon les sols et les milieux.

- Les pentes et les pénéplaines sableuses, qu'elles soient d'origine détritique ou éolienne (dunes fixées) évoluent à peu près de la même façon. Un pâturage intense entraîne une modification de la composition floristique du tapis herbacé, avec la prolifération de certaines espèces appétées comme Cenchrus biflorus ou Zornia glochidiata, qui présentent une certaine résistance à l'effet de pâture, ou inappétées comme Heliotropium strigosum. Parallèlement, on remarque presque toujours une diminution du couvert herbacé.

La mise en culture du sol et les sarclages entraînent une dénudation du sol qui l'expose à l'érosion éolienne en saison sèche. Le vent reprend les sables mal fixés et dessine des vaguelettes caractéristiques (ripple marks). L'extension du phénomène conduit à une remobilisation irréversible et la formation de dunes vives à l'aspect saharien (exemple d'Oursi). L'effet érosif des premières grosses pluies est considérable et les pentes mal fixées par une végétation discontinue ou exploitée à l'excès sont sujettes à une déflation en taches, formant des plages déprimées, en «coups de cuiller», caractéristiques.

- Les glacis recouverts d'un manteau sableux et les glacis sableux sont fragiles à l'érosion. Un couvert végétal insuffisant ne peut s'opposer au ruissellement qui emporte l'horizon superficiel meuble et fertile jusqu'à l'horizon sousjacent plus compact. Celui-ci finalement se trouve seulement recouvert par de petits bancs sableux en «microdunes», seuls points de fixation de la végétation herbacée.

- Les glacis limono-sableux réagissent d'abord au surpâturage par une diminution de la densité du tapis herbacé et l'extension des plages de sol nu aux endroits les plus érodables. Les eaux de pluie sont moins bien absorbées par le sol et le ruissellement, moins freiné par la végétation, érode les horizons superficiels en haut de pente, ce qui appauvrit le sol et dépose les éléments fins en bas de pente où les dessiccations successives forment un enduit superficiel qui colmate le sol et le rend physiologiquement sec.

Le tapis herbacé devient peu productif ou disparaît totalement. Les arbustes, mal alimentés en eau, réduisent leur couronne ou meurent. Le phénomène est particulièrement spectaculaire dans les «bush» installés sur sols squelettiques en raison du nombre d'arbres morts que l'on trouve actuellement.

- Les bas-fonds sont le plus souvent en très bon état puisque les eaux non infiltrées sur les versants s'y trouvent collectées et qu'elles reçoivent en plus les éléments chimiques lessivés. Une exploitation pastorale excessive entraîne cependant une dénudation et un colmatage du sol par plaques et la végétation ligneuse elle-même peut disparaître.

En somme, on constate une relation étroite et une interdépendance entre les modifications qui apparaissent au niveau du sol et l'évolution de la végétation. La disparition des horizons superficiels meubles faisant apparaître les horizons inférieurs plus compacts, la formation de croûtes superficielles peu perméables, l'alimentation rapide des eaux de pluie par ruissellement, tout cela s'oppose à une pénétration normale des eaux de pluie dans le sol. Certains glacis ne s'humidifient que sur 3 ou $4 \mathrm{~cm}$ après une forte pluie et s'assèchent donc très vite. Les réserves en eau à la surface sont très insuffisantes pour fournir aux jeunes plantules l'humidité nécessaire à la survie. Les réserves plus profondes se 
reconstituent très mal et les arbres, insuffisamment alimentés, disparaissent.

L'effet régulateur de l'alimentation hydrique du sol diminue avec l'érosion qui suit la disparition du couvert herbacé et n'est plus suffisante pour compenser les irrégularités des précipitations sahéliennes. De la sorte, le bilan hydrique des sols dégradés ressemble à celui des sols situés dans des conditions climatiques plus sévères et plus sèches et la végétation qui reste a des caractères sahéliens prononcés.

Quel que soit le type de milieu, la dégradation comprend une diminution de l'infiltration des eaux de pluie, donc la péjoration du bilan hydrique du sol et s'accompagne d'un ruissellement érosif accru. Le schéma de la figure 1 montre l'enchaînement des phénomènes sur l'ensemble d'un versant. On remarque l'intérêt de protéger en premier lieu le haut de pente, point de départ du ruissellement. On comprend aussi que la dégradation est plus forte en bas de pente et c'est là en effet que les phénomènes sont souvent les mieux développés.

\section{LES CAUSES}

Il n'est pas toujours facile de remonter jusqu'aux causes de la dégradation car elles sont multiples et généralement intriquées. Il est certain que l'augmentation de la charge en bétail depuis plusieurs décennies, à la suite de l'augmentation régulière de la population et des progrès en matière de santé animale, est une cause très importante de la dégradation de la végétation. La preuve en est l'état actuel de l'environnement à proximité de certains points d'eau permanents ou de certains centres où la surcharge animale est manifeste. Les agriculteurs passés et présents ont aussi leur part de responsabilité en raison de la fragilité à l'érosion de certains périmètres mis à nu par les défrichements et les sarclages et il est clair que la protection du milieu passe par des interdictions de mise en culture ou par la constitution de systèmes anti-érosifs efficaces parallèles à la mise en culture. Une autre cause de dégradation n'est pas maitrisable: c'est la sécheresse. Depuis 1971, certaines espèces graminéennes sud-sahéliennes ont disparu de la région de l'Oudalan ou sont devenues rares. $\mathrm{Ce}$ sont par exemple, Ctenium elegans, Elionurus elegans, Cymbopogon giganteus. Certains ligneux ont été sérieusement éprouvés: Pterocarpus lucens, Grewia spp., Commiphora africana. Les phénomènes d'érosion qui se manifestent depuis plusieurs années déjà ont amplifié les effets de la sécheresse sur les végétaux.

\section{SCHEMA D'UN TRANSECT EN COURS DE DEGRADATION}

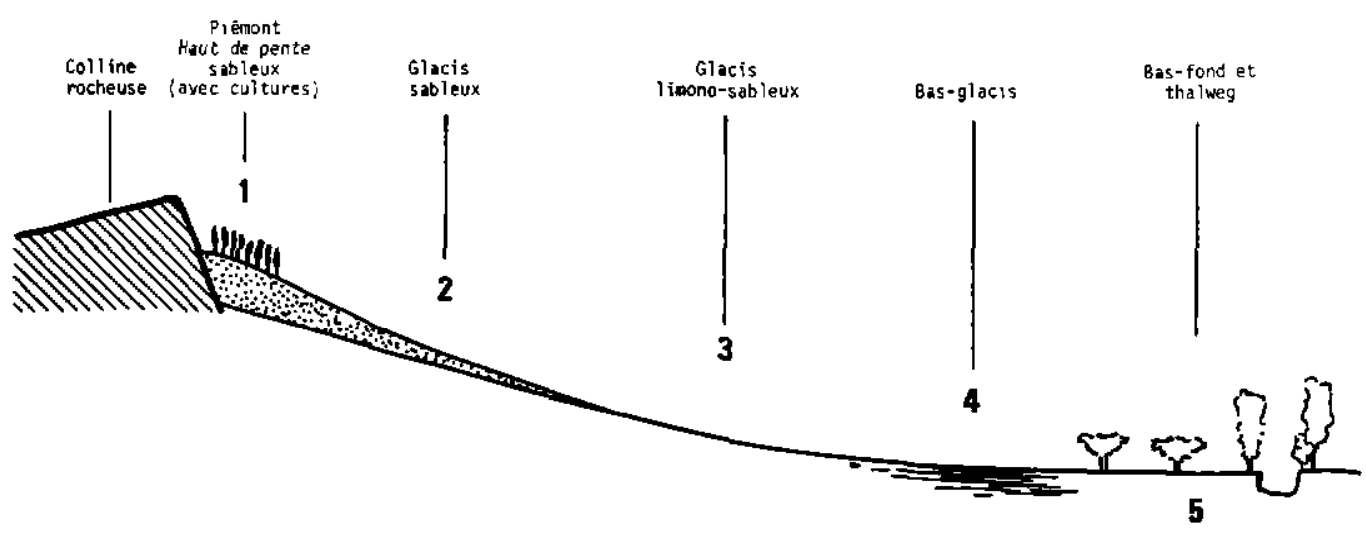

1. - Haut de pente

- Mise en culture et dénudation du sol

- Départ du ruissellement

- Plages de deflation, érosion en « coups de cuiller », apparition de ravines importantes.

2. - Glacis sableux

- Mises en culture ou surpâturage

- Dénudation du sol et alimentation du ruissellement

- Elimination partielle de l'horizon superficiel sableux et formation de « microdunes».

\section{3. - Glacis limono-sableux}

- Ruissellement intense et forte érosion superficielle

- Pénétration insuffisante de l'eau dans le sol

- Tapis herbacé éclairci, mort dé ligneux.

4. - Bas-glacis

- Dépôt des particules de sol, colmatage

- Disparition du tapis herbact, mort des ligneux.

5. - Bas-fond et thalweg

- Abondamment alimentés en eau

- Végétation en bon état. 


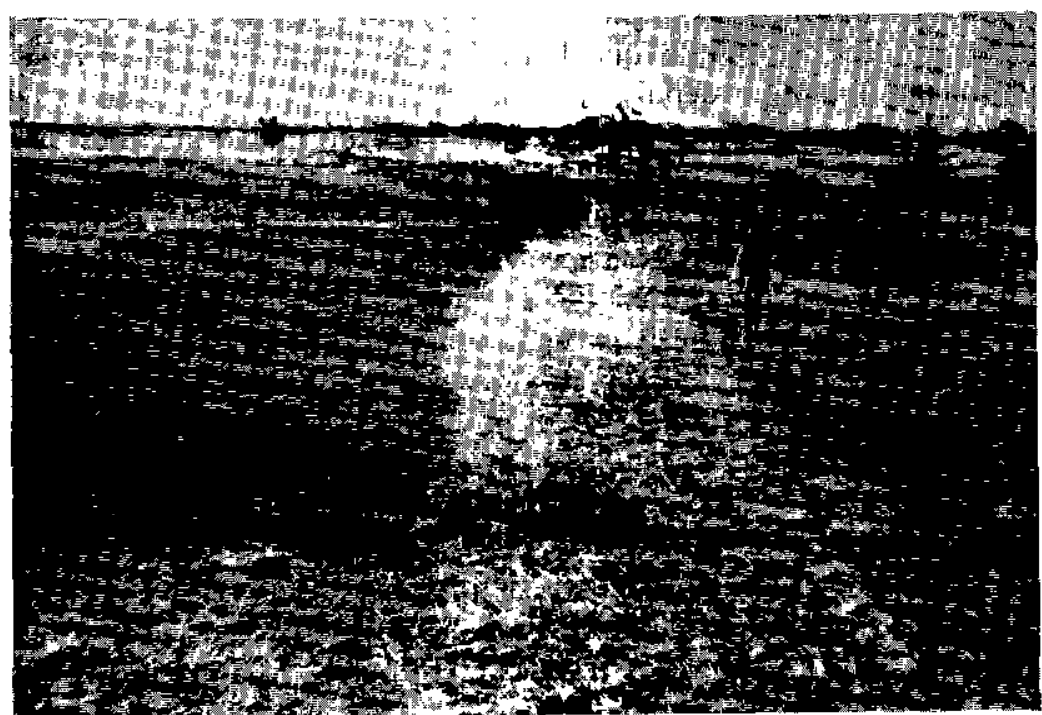

Photo 1. - Un travail superficuel très léger avec un rouleau cultipacker en début de saison des pluies a permis l'installation de graminées (Markoye. HauteVolta).

Photo 2. - Passages crolsés d'une sous-soleuse sur un bas-glacıs dégradé (GoromGorom, Haute-Volta).
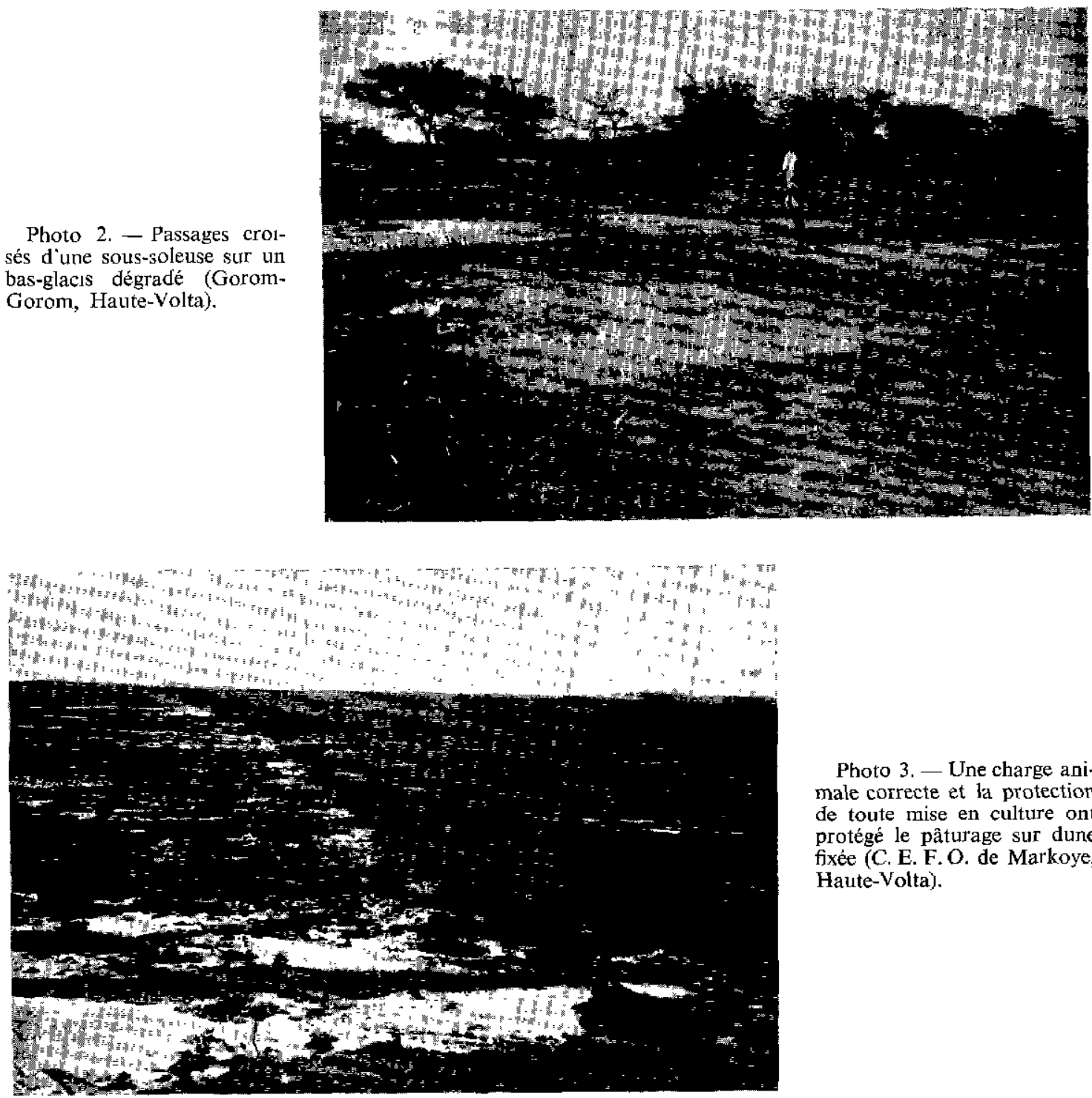

Photo 3. - Une charge animale correcte et la protection de toute mise en culture ont protégé le pâturage sur dune fixée (C. E. F.O. de Markoye, Haute-Volta). 
Photo 4. - En bas de pente, les éléments fins transportés par les eaux de ruissellement se déposent et forment une croûte imperméable et stérıle. Les arbustes meurent.

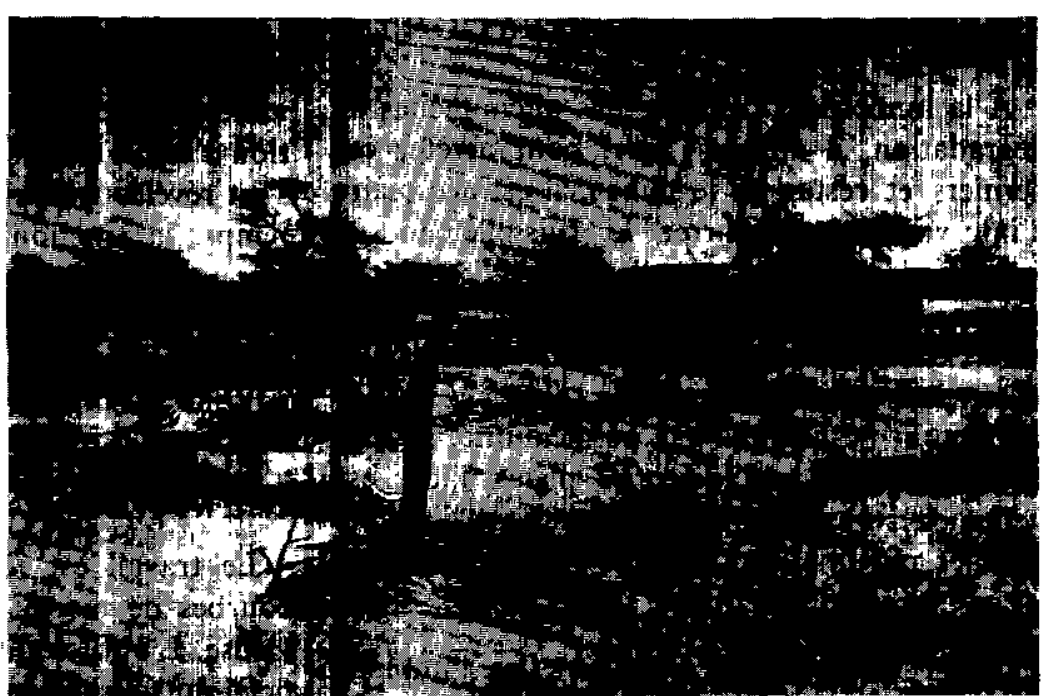

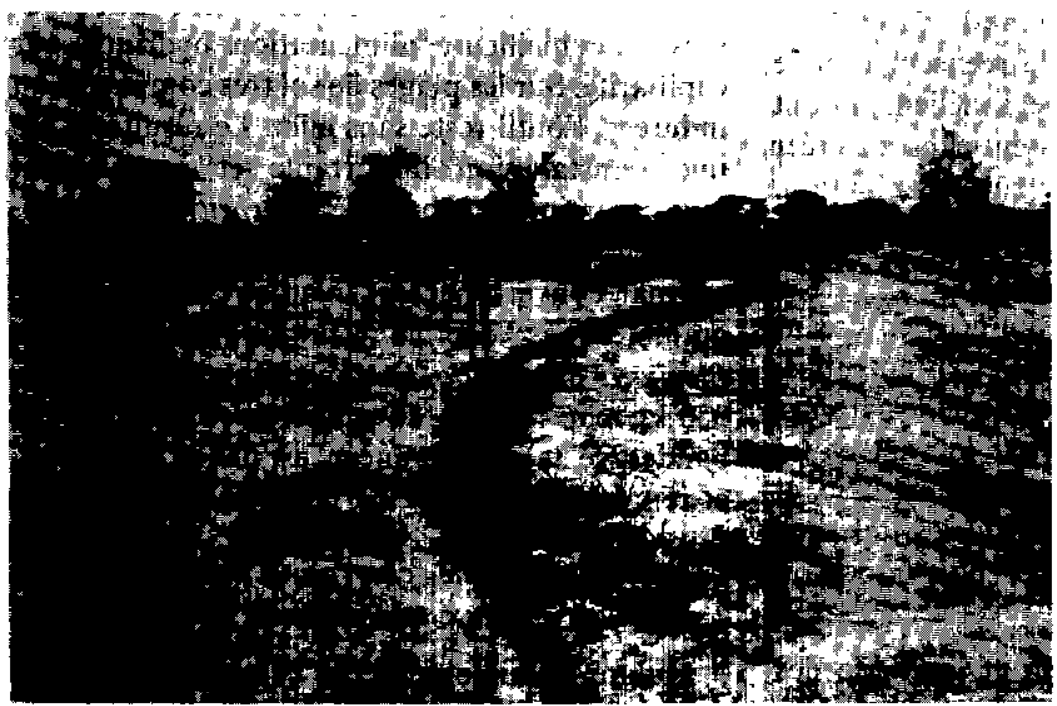

Photo 5. - Réapparition de l'herbe autour du sillon, après le passage d'une charrue à disque sur un glacis sableux très érodé (Markoye, HauteVolta). La pente, assez faible, va de droite à gauche.

Photo 6. - Réınstallation du tapis herbacé sur les bandes travaillées sans semis avec un cultipacker. Pente de dune érodée (Markoye, Haute-Volta).

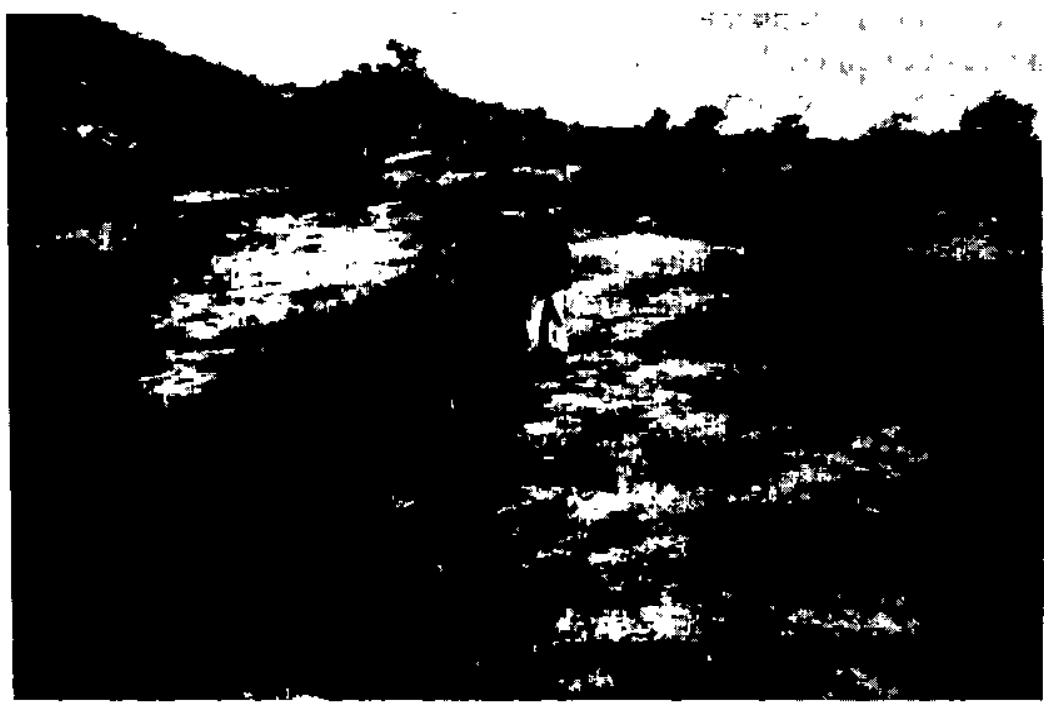




\section{LES ESSAIS DE RÉGÉNERATION}

A la lumière de l'analyse des phénomènes de dégradation, il apparaît que, pour arrêter ces derniers et restaurer le milieu, il est nécessaire d'intervenir pour barrer le ruissellement et favoriser la pénétration de l'eau dans le sol pour qu'une végétation herbacée et ligneuse réapparaisse. $\mathrm{Si}$ cette végétation persiste plusieurs années, on peut espérer que le sol se régénérera à son tout et gagnera en perméabilité. A voir l'herbe envahir les anciennes pistes, l'idée est venue de stimuler un nouveau départ du tapis herbacé par une simple action mécanique sur les premiers centimètres du sol.

L'effet recherché est de permettre l'implantation des graminées annuelles en brisant la croûte superficielle du sol au moment des premières pluies. Dans un grand nombre de situations, il n'est pas utile de réensemencer le sol car les diaspores, en zone sahélienne, sont très abondantes partout et l'essentiel est de créer des conditions favorables à leur germination. Dans ces essais, l'intervention mécanique devrait mettre le sol en état de :

- fixer les semences de graminées en créant des aspérités à la surface du sol et en ameublissant la terre. On observe en effet que les graines les plus fines sont déplacées par le vent et les eaux de ruissellement lorsque le sol est lisse, et qu'elles s'accumulent sur les obstacles, tels que banc de sable, touffe d'herbe, bois mort ou pierraille ;

- permettre la germination et la survie des jeunes semis. La nécessité des levées est menacée par la sécheresse entre deux pluies. C'est le sol qui doit alors maintenir les conditions d'humidité suffisantes. Dans le cas des sols croûtés en surface, on observe que l'eau pénètre très peu profondément après la puie et, par conséquent, la quantité d'eau stockée dans le sol est très rapidement évaporée. Lorsque la croûte superficielle a été détruite, le front d'humectation du sol est 2, 3, voire 4 fois plus profond. Dans ces conditions, le sol peut rester humide pendant plus longtemps ;

- assurer une croissance normale aux yégétaux. Au Sahel, la fertilité du sol est étroitement liée à la capacité de rétention en eau et au bilan hydrique. Un sol donné, avec la texture qui la caractérise, sera d'autant plus fertile que sa structure sera favorable à la constitution des réserves en eau.
Les essais de travail du sol ont été entrepris dans des milieux variés et avec des moyens variés, dans le Sahel de Haute-Volta. L'utilisation d'outils traditionnels et de l'énergie humaine s'est révélée très lente et pénible. La plupart du temps, la traction attelée ou le véhicule tout terrain ne sont pas assez puissants. Il est donc préférable de travailler avec un tracteur.

Voici les principaux essais et leurs résultats :

\section{Passage d'un rouleau type cultipacker sur les pentes sableuses dégradées.}

Le travail est réalisé approximativement en courbes de niveau au début de la saison pluvieuse. L'outil réalise un ameublissement superficiel du sol sableaux, mais sans l'émietter, et un léger tassement. Le résultat est une amélioration de la réaction du sol à la pluie et aux intervalles secs, une meilleure alimentation hydrique par capillarité. Sur les plages de sol très compactes ou indurées, l'outil reste sans effet. Le résultat a été une réinstallation importante et pérenne du tapis graminéen à base de Cenchrus biflorus (cf. cliché $\mathrm{n}^{\circ} 6$ ). Sur les espaces non travaillés, le sol est mal couvert par des plantes annuelles non graminéennes comme Zornia glochidiata ou Blepharis linariifolia. Cette végétation représente $350 \mathrm{~kg}$ par hectare de matières sèches. Sur les. bandes, le tapis graminéen est dense et continu et représente $1500 \mathrm{~kg}$ de matières sèches.

2. Passage d'une charrue à disques sur un glacis sableux très dégradé.

Malgré la faible pente de ce glacis, les pluies ont emporté l'horizon superficiel du sol et il ne reste qu'une surface compactée et lissée. De larges sillons espacés de 10 à $20 \mathrm{~m}$ ont été faits avec une charrue à disques, perpendiculairement au sens du ruissellement. La pénétration n'était pas très grande en raison de la compacité du. sol.

A l'endroit du passage de l'engin, un tapis herbacé irrégulier mais souvent dense, à base de Cenchrus biflorus et de Tragus spp., s'est installé (cf. cliché $n^{\circ}$ 5). Les eaux de ruissellement ont été en partie retenues par les sillons. On a noté une meilleure alimentation hydrique des plantes. et la durée de végétation s'est prolongée en début. de saison sèche. Les herbes restaient vertes sur les sillons quand toutes les graminées d'alentour étaient déjà fanées. De meilleurs résultats. auraient été acquis si le réseau de sillons avait été plus serré et avait été doublé de véritables. 
banquettes en haut de pente pour réduire les quantités d'eau de ruissellement. On peut prévoir de poursuivre un tel travail progressivement plusieurs années consécutives, en travaillant les interbandes de la même façon. 11 serait dangereux de faire un labour trop serré en une seule fois en raison des risques d'érosion.

\section{Passage de herse ou de cultipacker sur des glacis limono-sableux colluvionnés.}

Toute intervention superficielle qui brise la croûte superficielle permet une infiltration locale d'un peu d'eau, y provoque la germination et la survie des plantes. La herse doit être rigide, posséder peu de pointes et être chargée pour que les raies soient suffisamment profondes. Le cultipacker, sorte de rouleau constitué de lourds disques juxtaposés, à bords en triangle, brise, grâce à son poids et à son profil, la croûte superficielle du sol sur une série de lignes parallèles rapprochées. Sur certains glacis trop limoneux, les rayures ne sont pas suffisantes et disparaissent à la première pluie. Mais les résultats ont été le plus souvent extrêmement nets et là où le sol était primitivement pratiquement nu, un tapis de la graminée. Schoenefeldia gracilis s'installe au niveau des rayures. On observe aussi, juste sur les lignes, la germination de certaines espèces ligneuses. La production de fourrage sur les bandes travaillées n'est pas très élevée mais peut atteindre 200 à $400 \mathrm{~kg}$ de matière sèche par hectare. Cette installation ne paraît pas très persistante et la restauration de ce genre de pâturage sera entreprise sur plusieurs années en répétant chaque fois le travail en interbande. La protection, en particulier vis-àvis de chèvres, est nécessaire si l'on veut permettre la régénération des ligneux.

\section{Sous-solage des glacis limono-sableux éro- dés}

Divers glacis très érodés et dégradés, pratiquement dénués de végétation ont été sous-solés, tantôt en lignes croisées espacées de 5 à $10 \mathrm{~m}$, tantôt en lignes parallèles perpendiculaires à la pente et espacées de 10 à $20 \mathrm{~m}$. En haut de pente, où le sol est très érodé l'effet d'amélioration n'est pas sensible. Sur les bas de pente colluvionnés, les raies de passage de la soussoleuse sont couvertes d'herbe sur une largeur de $50 \mathrm{~cm}$. Le sol habituellement physiologiquement $\mathrm{sec}$, est humide sur $20 \mathrm{~cm}$ de chaque côté de la raie jusqu'à la profondeur de travail. Le volume de terre ainsi humidifié est donc faible. Le résul- tat le plus intéressant est la levée sur la ligne de très nombreux jeunes arbustes (Acacia, Balanites, Ziziphus). Ce genre de travail suivi d'une protection, surtout vis-à-vis des chèvres, pendant deux ans au minimum est à envisager pour une régénération de la strate ligneuse.

\section{Travail superficiel des colluvions de bas de pente}

Le labour superficiel des zones colmatées en bas de pente par les dépôts d'éléments fins a été essayé. La surface travaillée s'est irrégulièrement couverte d'herbe et le gain de fourrage représente 300 à $400 \mathrm{~kg}$ de matière sèche par hectare. Contre la parcelle, une zone de rétention des eaux de ruissellement s'est créée, large de quelques mètres. Le tapis de Schoenefeldia gracilis représente $2000 \mathrm{~kg}$ de matières sèches par hectare. La zone témoin est restée pratiquement nue. Un réseau serré de sous-solage dans ce type de sol a donné des résultats approchants et la moitié de la surface du sol s'est couverte de graminées.

Il ne paraît donc pas nécessarre que les sillons soient jointifs. Il semble suffisant, sur ces colluvions, de réaliser seulement des bandes travaillées de plusieurs mètres de large, perpendiculaires à l'axe de ruissellement.

\section{Essais de semis simultané de plantes four- ragères}

Des espèces fourragères ont été semées au moment du travail du sol, principalement Stylosanthes humilis et Cenchrus ciliaris. Les résultats sont décevants et les espèces spontanées apparaissent toujours les plus vigoureuses. Les graines des espèces spontanées, herbacées ou ligneuses, adaptées aux sols que l'on travaille, semblent présentes le plus souvent en nombre suffisant. Si l'effet du travail est durable, le ressemis s'opère automatiquement.

\section{CONCLUSION}

Un travail léger du sol peut être pratiqué pour restaurer les parcours sahéliens très dégradés. Il permet habituellement la formation d'un tapis graminéen à l'emplacement du passage des outils avec un supplément de production localisé de 20 à plus de $100 \mathrm{~g}$ de fourrage sec au mètre carré, selon les types de sol et d'intervention.

Une opération de régénération d'une zone pourrait se réaliser de la façon suivante : 
- d'abord atténuer ou éliminer les principales causes de dégradation. Pour beaucoup de pâturages, il s'agit de protéger du surpâturage ou du pâturage trop précoce et si l'on désire une régénération ligneuse, il faut faire une mise en défens pendant 2 à 3 ans, notamment vis-à-vis des chèvres;

- travailler le sol avec une méthode appropriée en bandes approximativement perpendiculaires à la pente. Ces bandes ne seront pas trop rapprochées de façon à ne pas créer des départs d'érosion et elles bénéficieront de l'apport de semences des pentes intermédiaires, suffisant pour former un tapis assez dense ;
- le travail sera poursuivi en interbandes pendant plusieurs années, de façon à augmenter peu à peu la surface travaillée.

\section{REMERCIEMENTS}

Nous tenons à remercier M. A. CIRE BA, Chef du Service de la Recherche Animale à la Direction de l'Elevage de Ouagadougou et M. A. LE MASSON, du C. I. D. R. à GoromGorom, pout leur collaboration et les féliciter pour leurs initiatives à propos des essais de travail du sol.

\section{SUMMARY}

Trials on the mecanical improvement of some sahelian deteriorated rangelands

Overstocking and the recent drought have led to a severe deterioration of some Sahelian rangelands of Upper-Volta, leading to a more or less acute disappearance of grazable vegetation. Partial protection improves pastures located on the more fertile soils. On the poorer soils light techniques such as harrowing and superficial tillage of the soil were tried with succes, but yield improvement remained slight.

\section{RESUMEN}

Ensayos de regeneración mecanica de algunos pasturajes sahelianos degradados

Se ocurre en ciertas regiones del norte de Alto-Volta, muy pobladas por el ganado, una degradación importante de la vegetación saheliana, sobretodo desde la sequedad, teniendo por consecuencia la disminución de las potencialidades pastorales y amenazando el mismo medio.

Se analizaron las principales formas de dicha degradación. Se ensayó regenerar los terrenos desnudados en diferentes condiciones sea por trabajos superficiales del suelo, sea por la acción de un arado de subsuelo, para mejorar el balance hidrico y permitir la réinstalación de la cubierta vegetal.

\section{BIBLIOGRAPHIE}

1. BOUDET (G.). Mali, Regional study and proposal for development. Ecol. Bull., Stockholm, 1976, 24 : 137-53.

2. BOUDET (G.). Les pâturages sahéliens, les dangers de dégradation et les possibilités de régénération; prıncipes de gestion amélıorée des parcours sahéliens. Rome, F. A. O./I. E. M. V. T., 1976. 104 p.

3. TOUTAIN (B.). Notice de la carte des ressources fourragères au $1 / 50000^{\mathrm{c}}$. Action complémentalre concertée D. G. R. S. T./O. R. S. T, O. M./G. E. R.D. A. T./I. E. M. V. T., 1976.61 p., 1 carte. 